\title{
Sentimento e emoções de homens no enquadramento da doença Covid-19
}

\author{
Men's feelings and emotions in the Covid-19 framing
}

\author{
Anderson Reis de Sousa (https://orcid.org/0000-0001-8534-1960) ${ }^{1}$ \\ Evanilda Souza de Santana Carvalho (https://orcid.org/0000-0003-4564-0768) ${ }^{2}$ \\ Thiago da Silva Santana (https://orcid.org/0000-0003-0987-0814) ${ }^{2}$ \\ Álvaro Francisco Lopes Sousa (https://orcid.org/0000-0003-2710-2122) ${ }^{3}$ \\ Thiago Fonseca Geanizelle Figueiredo (https://orcid.org/0000-0003-1024-8782) ${ }^{1}$ \\ Oscar Javier Vergara Escobar (https://orcid.org/0000-0003-3158-9017) ${ }^{4}$ \\ Tilson Nunes Mota (https://orcid.org/0000-0001-5836-2360) ${ }^{1}$ \\ Álvaro Pereira (https://orcid.org/0000-0003-1615-5528) ${ }^{1}$
}

\begin{abstract}
Objective: to understand how men's feelings and emotions contribute to the Covid-19 framing in Brazil. Method: Asocial-historical, qualitative study, carried out with 200 men resident in Brazil, through online search on digital platform. The grasped data were analyzed by the Collective Subject Discourse method in the light of the reference of epidemic disease proposed by Charles Rosemberg. Results: Negative feelings and anxiety prevailed due to the knowledge about the growing number of hospitalized patients and deaths from the pandemic conveyed in the news. For men, the optimism is necessary to encourage attitudes with responsibility and trust that the crisis will be overcome.Subsequently, men present a set of attitudes and behaviors for coping with the pandemic. Moreover, the acceptance signals the emergence of the fourth dramaturgical act of the Covid-19framing. Conclusion: Men's feelings and emotions, in this historic context, pervade three of the four acts of the Covid-19 framingin Brazil.
\end{abstract}

Key words Pandemics, Coronavirus infections, Men's health, Masculinity, Delivery of health care
Resumo O objetivo deste artigo é compreender como os sentimentos e as emoções de homens contribuem para o enquadramento da doença Covid-19 no Brasil. Estudo sócio-histórico, qualitativo, realizado com 200 homens residentes no Brasil, mediante pesquisa online em plataforma digital. Os dados apreendidos foram analisados pelo método do Discurso do Sujeito Coletivo à luz do referencial de doença epidêmica proposto por Charles Rosemberg. Prevaleceram sentimentos negativos e ansiedade como consequência do conhecimento acerca do crescente número de hospitalizados e mortos pela pandemia veiculada nos noticiários. Para os homens, o otimismo é necessário para encorajar atitudes com responsabilidade e confiar de que a crise será superada. Na sequência os homens apresentam um conjunto de atitudes e comportamentos para o enfrentamento da pandemia. E, a aceitação sinaliza a emergência do quarto ato dramatúrgico do enquadramento da Covid-19. Os sentimentos e as emoções de homens, no presente contexto histórico, atravessam três dos quatro atos de enquadramento da Covid-19 no Brasil. Palavras-chave Pandemias, Infecções por coronavírus, Saúde do homem, Masculinidade, Assistência à saúde 


\section{Introdução}

A pandemia da COVID-19 configura-se como o maior desafio sanitário do século 21 , com mais 6.287.771 casos confirmados em todo o mundo e mais de 375 mil mortes em 01 de junho ${ }^{1}$. Sendo esse um vírus novo, com alta taxa de transmissibilidade, não foi possível determinar medidas de contenção efetivas e, com isso, houve rápida disseminação por diversos países alcançando o status de pandemia. Essa situação provocou múltiplos impactos, afetando a população mundial tanto no âmbito individual quanto no coletivo e sendo considerado uma Emergência de Saúde Pública de Importância Internacional (ESPII). Desse modo, no que concerne à dimensão coletiva, a pandemia tem sido degradante nas esferas social, política, econômica e educacional ${ }^{2}$, mobilizadoras de múltiplos sentimentos e emoções humanas.

No atual contexto pandêmico o Brasil tem sofrido significativamente com os impactos provocados pela doença somados à crise política $\mathrm{e}$ governamental ${ }^{3}$. Em 23 de maio de 2020 o Brasil ocupava a segunda posição em distribuição de casos da Covid-19 entre os países com maior número de casos. Até a presente data o Brasil já registrava mais de 346.398 casos da doença. Desse total, $22.013(6,3 \%)$ evoluíram a óbito, $182.798(52,6 \%)$ estavam em investigação e $142.587(41,0 \%)$ já haviam apresentado cura da doença. Quando comparado com o dia anterior, o país registrou 965 novos óbitos, perfazendo um incremento de 4,6\% (965/21.048). Em apenas 21 semanas epidemiológicas, aproximadamente 17.000 pessoas foram a óbito 4 .

Por se tratar de um fenômeno sócio-histórico de interesse global, o contexto pandêmico emergido com o surgimento do SARS-CoV-2 tem sido fator influenciador de modificações estruturais nas relações e nas organizações humanas em todos os países, em especial naqueles em desenvolvimento ${ }^{5}$. Essa nova história tem afetado os fluxos migratórios, as cadeias de produção, as importações e as exportações, as relações internacionais existentes e os modos de subsistência, o que gera grande preocupação, sobretudo no que se refere ao alcance da agenda 2030 e os 17 objetivos do milênio para o desenvolvimento sustentável coordenados pela Organização das Nações Unidas $(\mathrm{ONU})^{4}$.

As doenças suscitam a elaboração de narrativas sócio-históricas, válidas para um determinado tempo histórico, e produzem representações e imagens sobre o evento pandêmico que no geral são associados a acontecimentos assustadores, trágicos e desagregadores do convívio social. A partir de pandemias como a da gripe espanhola (1918) e da influenza H1N1 (2009), foram observados que novas representações e dialéticas são postas em jogo e a abordagem sócio-histórica se apresenta como capaz de explicar a complexidade de acontecimentos historicamente demarcados, bem como as ideias que circulam entre os grupos e modelam seus comportamentos, ademais de favorecer a compreensão da dinâmica afetiva permitindo a análise de sentimentos e emoções ${ }^{6}$.

Fundamentado no pensamento do construcionismo social o historiador social da medicina/saúde Charles Rosenberg teoriza acerca dos processos pandêmicos. Sobre essa premissa, o estudioso sob um olhar historiográfico e multidimensional desenvolve uma análise da percepção sobre as mudanças sociais geradas por doenças, em especial, das epidemias e das pandemias, teorizando o que denominou de enquadramento ${ }^{6}$.

A doença, para esse autor, é entendida como produto socialmente construído conforme esquemas que procuram explicá-la e classificá-la de modo organizado como numa "moldura" ou um "quadro" e implica na articulação entre individualidade, negociações, diagnóstico social, unidade e diversidade. O processo de enquadramento é cíclico e apresenta-se em atos característicos que se iniciam pela "negociação", perpassa pela "ressignificação" e finaliza com o "esquecimento" ".

Dados globais revelam as preocupações e os sentimentos experienciados pela população face ao acesso às notícias, medidas sanitárias para evitar a propagação do vírus que implicaram em mudanças no cotidiano e nas interações derivadas da pandemia ${ }^{8-10}$. Estudo aponta que não existe seletividade para o contágio do novo Coronavírus, entretanto a doença irá repercutir de forma diferente em razão de marcadores de gênero, raça e classe ${ }^{11}$, que se interseccionam como marcadores de diferenciação social, permeando a construção das masculinidades ${ }^{12}$.

A crescente morbimortalidade de homens pela Covid 19 tem sido explicada por fatores genéticos, comportamentais e de estilo de vida ${ }^{13-15}$. Situações desfavoráveis para a saúde de homens tem sido observada em cenários com baixa procura por serviços de saúde na Atenção Primária $^{16}$. No Brasil, o expressivo número de óbitos, internações hospitalares e complicações graves causadas pela Covid-19 em homens suscita que às masculinidades, comportamento social, estilo de vida, sejam considerados nos estudos sobre o enquadramento da pandemia ${ }^{17}$. 
Partimos do pressuposto de que a pandemia deflagra, nos homens, sentimentos e emoções que fazem parte dos processos de enquadramento sócio-histórico da doença. Assim este artigo foi guiado pela seguinte questão: Como homens residentes no Brasil expressam sentimentos e emoções e contribuem para o enquadramento da doença Covid-19? Para responder essa questão, este estudo tem o objetivo de compreender como os sentimentos e as emoções de homens que contribuem para o enquadramento da doença Covid-19 no Brasil.

\section{Método}

Estudo sócio-histórico ${ }^{18,19}$, qualitativo, realizado em ambiente virtual com homens residentes no Brasil, de identidade de gênero cisgênera, transgênera e não binária. A pesquisa foi operacionalizada durante o período de quarentena e distanciamento social determinado pelas autoridades sanitárias na maior parte do país, em virtude da pandemia da Covid-19.

Foram recrutados homens mediante convite e disponibilização de um link através de $e$-mails, convites inbox Facebook, Instagram e Whatsapp. Para alcance dos participantes adotou-se a estratégia de recrutamento consecutivo Snowball $20^{20}$ e a coleta foi encerrada por conveniência ao alcançar 200 homens que assinalaram o termo de consentimento livre e esclarecido.

A coleta de dados foi realizada entre março e maio de 2020, e o material empírico produzido através de um formulário semiestruturado hospedado em uma plataforma digital e gratuita, na interface Google Forms e disponibilizado nas redes sociais Facebook, Instagram e Whatsapp vinculadas ao grupo de pesquisa. Adotou-se critérios de segurança de proteção dos dados e seguiu-se os critérios do Revised Standards for Quality Improvement Reporting Excellence - SQUIRE 2.0 para garantia do rigor metodológico.

O formulário foi composto por blocos de questões: 1 - aspectos de caracterização sociodemográfica, laboral e de saúde; 2 - aspectos relacionados às atitudes e estratégias de enfrentamento da pandemia; e 3 - abordou emoções e sentimentos emergidos durante pandemia no Brasil. Os resultados apresentados neste artigo exploraram as respostas do bloco de questões 3.

Para alcance da qualidade da pesquisa, adotou-se o protocolo Consolidated Criteria for Reporting Qualitative Research - COREQ. No que tange à formação e qualificação da equipe, esta foi composta por quatro doutores, dois mestres e dois graduandos. $\mathrm{O}$ formulário foi autoaplicado, seu preenchimento durou de 20 a 30 minutos e as respostas foram extraídas e transcritas para documento em Word.

Para determinar a saturação teórica dos da$\operatorname{dos}^{21}$, a equipe de pesquisa realizou três rodadas de discussão dos temas obtidos nos discursos e reproduzidos dentre os participantes e observando as figuras derivadas da triangulação de estratégias de análise, obtidas dos Softwares NVIVO12 e Iramutec.

Este estudo atendeu às recomendações da Resolução 466/2012 que versa sobre os aspectos éticos na pesquisa com seres humanos, em todas as fases, e foi aprovado pelo Comitê de Ética em Pesquisa.

A análise seguiu as etapas do método do Discurso do Sujeito Coletivo (DSC): transcrição na íntegra dos depoimentos; separação dos fragmentos dotados de sentido; organização dos conjuntos de fragmentos; identificação das Expressões Chave (EC) ou elementos figurativos; junção das EC, buscando a reconfiguração de um discurso único, composto por pensamentos individuais de sujeitos de um mesmo grupo. $\mathrm{Na}$ sequência os pesquisadores avaliam e validam as Ideias Centrais (IC), redigem e constroem os discursos-sínteses, nomeando-os, ou seja, atribuindo-lhes o título do Discurso do Sujeito Coletivo ${ }^{22}$.

Segundo Lefevre, os discursos-sínteses, que expressam o pensamento social, representados por quatro IC apresentadas nos resultados deste estudo. Em íntima articulação o DSC faz interface com a perspectiva sócio-histórica em investigação qualitativa, na medida em que se dedica em expressar opiniões de uma coletividade, além de deparar-se com a observação e a análise de distintos discursos verbais e gestuais ${ }^{22}$. Nesse sentido, o enfoque e o direcionamento sócio-histórico e das bases epistêmicas do DSC estão direcionados à compreensão singular da relação do indivíduo e da totalidade, possibilitando refletir e refratar a realidade e a tessitura da vida social ${ }^{18,23}$.

Considerando a necessidade de analisar teoricamente os achados à luz da pespectiva sócio -histórica, e reconhecendo a vasta contribuição aportada por Charles Rosenberg nesse campo do saber, o corpus foi submetido à interpretação suportada na obra: Explaining epidemics and other studies in the history of medicine que permitiu enquadrar a doença epidêmica em quatro atos representativos, a saber: revelação progressiva, gerenciamento da aleatoriedade, negociação da resposta do público e subsidência e retrospecção, a 
fim de evidenciar o surgimento de características definidoras e elementos próprios em contextos de epidemias e pandemias ${ }^{6}$.

\section{Resultados}

Os participantes encontravam-se na faixa de 18 a 67 anos, dentre eles $93,6 \%$ se autodeclararam cisgêneros, $47,7 \%$ gays, $38,7 \%$, pardos e $22,1 \%$ pretos, $66,7 \%$ cursaram o ensino superior completo, $72,3 \%$ residiam em estados do Nordeste, $21,95 \%$ conviviam com familiares não idosos, $67,45 \%$ dos respondentes residiam em casas de alvenaria, e $33,2 \%$ viviam com renda aproximada de cinco salários mínimos.

Os sentimentos e as emoções deflagrados em maior expressividade pelos homens durante a vivência da pandemia, encontram-se evidenciados na Árvore de Similitude na Figura 1.

Observa-se uma árvore com quatro ramificações mais salientes e de fortes conexões com seus ramos. Estas evidenciam no centro a "ansiedade", mobilizada por "apreensão", "insegurança", "inquietação", "instabilidade" e "medo" que se conecta com todas as ramificações representadas no ramo superior à direita por "preocupação", e demarcam o primeiro e o segundo ato.

O gráfico leva a inferir que o enquadramento da Covid-19 a partir do discurso masculino encontra-se focado no segundo ato, estando a "ansiedade" como consequência do conhecimento acerca do crescente número de hospitalizados e mortos pela pandemia veiculada nos noticiários.

No ramo superior esquerdo aparecem palavras que representam sentimentos positivos, atitudes e estratégias de enfrentamento face à pandemia. Para os homens, estar "otimista" é necessário para se "encorajar" a tomar posição de atitudes com "responsabilidade", nesse sentido pode-se obter a "confiança" de que a crise pode ser superada. Este ramo se aproxima do terceiro ato do enquadramento da doença pandêmica, enquanto estágio em que os homens apresentam à sociedade um conjunto de atitudes e comportamentos para o seu enfrentamento.

A "aceitação" emerge discretamente, em ramo único, que sinaliza para o surgimento de uma pequena movimentação para a aparição do quarto ato dramatúrgico do enquadramento da Covid-19 a partir da experiência masculina no Brasil.

A reunião de discursos-sínteses pelos homens desvela sentidos semelhantes e/ou complementares acerca dos sentimentos e emoções face a pandemia em seu país de residência. $\mathrm{O}$ aparecimento desses sentimentos se organiza por meio de quatro Ideias Centrais de análise, descritas a seguir.

\section{Ideia central A: ato 1 - da negação ao progressivo reconhecimento}

O primeiro ato característico do enquadramento da Covid-19 está permeado pelo temor gerado pela incapacidade do Estado em gerir o enfrentamento da pandemia no Brasil. O discurso masculino evidenciou ainda a preocupação e o receio de que haja um colapso no sistema de saúde brasileiro em razão da pandemia do novo Coronavírus.

Logo no início do surgimento da pandemia eu não acreditava muito e não realizava as ações que estavam sendo recomendadas, pois imaginava que não era grave e que não iria chegar aqui nem tão pouco me atingir. Mais adiante eu passei a me preocupar e temer os aspectos externos, como, por exemplo, a fragilidade do governo em enfrentar a pandemia no país. Sinto-me apreensivo com a falta de testes rápidos, com o tipo de atenção que está sendo destinada aos profissionais de saúde para que possam atuar com segurança e a capacidade de lidar com os problemas que possam surgir durante e após a pandemia. Uma das minhas grandes preocupações é que não haja serviços de saúde pública disponiveis para atender as pessoas com a COVID-19, mas às vezes por eu presenciar tantas divergências políticas e governamentais apresentadas na mídia, eu começo a ter dúvida se de fato a doença é tão grave mesmo. Tenho receio que aconteça a situação que a Itália está passando e por saber que já temos um país gigantesco, desigual e com muitos problemas que afetam o SUS eu passo a me preocupar com um possivel colapso no sistema de saúde. (DSC de homens residentes no Brasil durante a pandemia da Covid-19).

\section{Ideia central B: ato 2 - percepção do problema, aceitação, explicação com base nos valores}

Em razão da preocupação com o aumento da mortalidade pela Covid-19 no Brasil, o discurso coletivo de homens expressa tensão, medo e dor existente face à vivência do contexto pandêmico, momento em que já é possível constatar a presença da doença instalada, representando o segundo ato de enquadramento. Nota-se o exercício da compreensão do fenômeno e a comoção frente ao cenário presenciado.

Outro fator que tem aumentado a minha tensão e o medo é o surgimento dos primeiros óbitos 


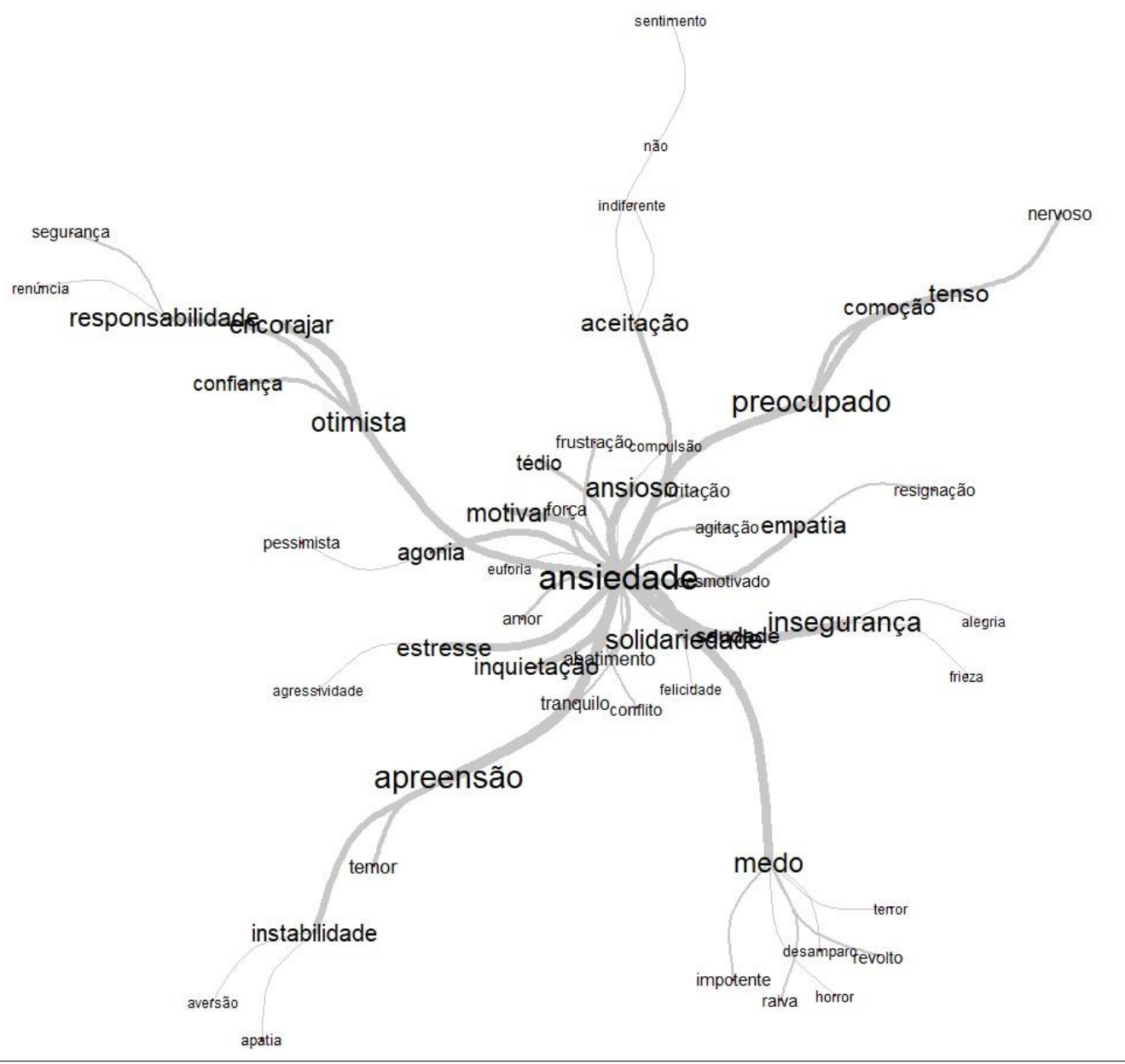

Figura 1. Árvore de Similitude acerca dos sentimentos e emoções deflagrados de homens face à pandemia, Salvador, Bahia, Brasil, 2020.

Fonte: Dados da pesquisa. Salvador, Bahia, Brasil, 2020.

de pessoas causados pela COVID-19 no meu estado. Antes eu via apenas pela televisão e agora eu já percebo que a situação é um problema real e já está afetando a minha localidade, assim como tem acontecido em outros estados. A todo momento eu tenho sido informado sobre estudos realizados por cientistas e isso tem me levado a um processo de reflexão para poder me preservar contra o vírus. Presenciar esse cenário me deixa apreensivo e às vezes aterrorizado, pois eu temo que possa acontecer o pior e que possa haver muitas mortes no país. Além disso, também me dói o fato de ter que presenciar a dor do outro e não poder ajudar. (DSC de homens residentes no Brasil durante a pandemia da Covid-19).

\section{Ideia central C: ato 3 - negociação com respostas, adesão e não adesão de medidas}

Motivados pela insegurança, medos e incertezas além da ruptura das interações sociais, emergiu-se preocupações expressivas que se traduzem em atitudes na tentativa de enfrentar os impactos provocados pela Covid-19 no país.

Tenho estado muito preocupado. O fato de ter que ficar isolado e não poder sair, o que tem sido difícil de enfrentar, afinal de contas como os demais brasileiros eu sinto muita vontade de realizar as minhas atividades normais e estar no ambiente externo. Fico inseguro com o fato de não saber até quando terei que ficar em isolamento. Além disso, em razão do prolongamento da quarentena eu tenho temor de que haja um prejuízo nas relações 
sociais, como, por exemplo, na elevação de conflitos conjugais, separação de casais, afastamento de amigos e do convívio social e com isso gerar um impacto na sociedade brasileira. Como consequência de todas essas situações que eu tenho vivido, eu tenho buscado seguir as recomendações do Ministério da Saúde e dos profissionais de saúde e cientistas que estão sempre apontando nos programas de televisão e na internet, e tenho buscado também realizar atividades que diminuam o estresse, a ansiedade, a solidão, a incerteza e o medo. (DSC de homens residentes no Brasil durante a pandemia da Covid-19).

\section{Ideia central D: ato 4 - retrospecção/reflexão que se constrói a partir da experiência}

Considerando que a investigação se deu no curso da progressão epidêmica da Covid-19 no Brasil, o processo de enquadramento da Covid-19 a partir dos discursos dos homens não se mostrou tão consolidado. Tal cenário pode se dar em razão da não aceitação completa da doença por parte desse grupo. No entanto, em alguns discursos percebe-se indícios deste ato, a exemplo da expressão "aceitação" apontando para um novo ramo da árvore de similitude, o que significa que este ato poderá ser alcançado, no qual se constitui a partir da experiência e da busca por extrair lições a serem apreendidas pelo fenômeno.

Tenho buscado aprender com toda essa situação e entender que se trata de algo passageiro. Busco realizar novas atividades e desenvolver novos aprendizados, tentando tirar proveito desse momento difícil. É um momento que eu tenho refletido ao meu respeito, sobre determinados grupos sociais e a respeito da humanidade, reconhecendo o quanto somos vulneráveis e frágeis. (DSC de homens residentes no Brasil durante a pandemia da Covid-19).

\section{Discussão}

Coletar, relatar e analisar dados da Covid-19 de maneira desagregada por sexo e responsiva ao gênero é uma meta da Organização Mundial da Saúde para garantir que as respostas dos países sejam sensíveis ao gênero. No entanto, nada foi publicado até o momento na literatura latino-americana, embora dados epidemiológicos estimam, em um futuro próximo, as Américas como o novo epicentro da pandemia. Nesse sentido, este manuscrito é pioneiro ao abordar os senti- mentos experienciados por homens brasileiros face à pandemia da Covid-19.

O enfrentamento de uma pandemia depende entre outras ordens, da ação do Estado para gerir as melhores e as mais coerentes estratégias com fins na diminuição de casos novos e/ou eliminação da transmissão. Por outro lado, problemas de caráter governamental têm sido evidenciados, países permeados por conflitos partidários, divergências ministeriais, contraposição ou negacionismo ao conhecimento científico e o distanciamento das medidas sanitárias recomendadas pelas autoridades nacionais e internacionais. Tal modelo de masculinidade expresso pela figura presidencial que inspira a invencibilidade ao vírus, a minimização dos agravos, o descumprimento das medidas sanitárias e de prevenção, de certo modo podem influenciar na compreensão, na aceitação e na tomada de decisão dos homens no enfrentamento à pandemia, merecendo ser levadas em consideração.

Cenário como este tem sido identificado sócio-historicamente, como foi observado com o surgimento do HIV, durante a epidemia da gripe espanhola, da febre amarela e do $\mathrm{H} 1 \mathrm{~N} 1^{6}$. $\mathrm{Na}$ contemporaneidade, em países como o Brasil, é possível observar a polarização político-partidária e ideológica na centralidade das informações sobre o contexto pandêmico. Essa postura gera repercussões sociais que passam a comprometer o bem-estar social e psicológico da população, como ocorre no público masculino. Além disso, as pandemias anteriores foram enquadradas de modo a observar as semelhanças, as diferenças e as atualizações evidenciadas no discurso masculino sobre a Covid-19, a fim de potencializar a compreensão sócio-histórica da doença na contemporaneidade ${ }^{7}$.

Sob este aspecto, este estudo revela por meio do discurso que os homens sofrem repercussões de caráter negativo decorrente do temor experienciado pela percepção de incapacidade por parte do governo no enfrentamento da pandemia no Brasil ${ }^{24}$. Os fragmentos discursivos estão mais aparentes no primeiro e no segundo ato do enquadramento da Covid-19 no Brasil. Importa destacar que as preocupações expressas nos discursos perpassam por um processo de construção de um diagnóstico social que é instituído a partir da inscrição social desses sujeitos, que se articulam em dada unidade e diversidade, como forma de reagir ao fenômeno, de pensar e tecer considerações sobre o mesmo e de tomar decisões ${ }^{6}$.

Ao perceberem os conflitos das autoridades políticas e sanitárias do Brasil, para o controle 
e a disseminação da doença epidêmica no país, os homens se sentem preocupados, apreensivos, descontentes e descrentes, o que pode prejudicar a aceitação da pandemia, que se espera que ocorra no segundo ato, e assim impede o seu progresso para os atos subsequentes.

A existência de incerteza e insegurança por parte da ação do Estado na governabilidade das ações proporciona a elevação do estresse, da ansiedade e do medo, sendo estes, expressivos complicadores no enfrentamento do problema ${ }^{24,25}$. Observa-se ainda que o discurso masculino desvela preocupação para com a integridade humana dos profissionais de saúde, para com a atenção frente à COVID-19, situação que reflete o panorama nacional que expressa um número elevado de profissionais mortos, adoecidos e afastados pela doença no país. Além disso, percebe-se no discurso masculino que se deu no primeiro ato, a presença de narrativas individualistas, em que os homens narram pouco sobre o coletivo. Explicitam preocupações de ordem pessoal e profissional, relacionadas ao trabalho, manterem-se ativos, às projeções sociais e de maneira discreta a dimensão da família e das redes de afeto, o que pode ter raiz na construção das masculinidades.

Acredita-se que essa repercussão social advinda do surgimento da pandemia da Covid-19 Brasil em associação com a incapacidade do governo em enfrentar a situação, esteja também motivada pelo processo de degradação do Sistema Único de Saúde, do desmantelamento da aparelhagem pública, do retrocesso no avanço da ciência e tecnologia e da corrupção que assola o país inclusive durante o período da pandemia ${ }^{26}$.

Desse modo, o ato de se preocupar com este aspecto emergido com a pandemia do novo Coronavírus tem relação com o entendimento dos homens estudados sobre a experiência em relação à doença. Para tanto, são incluídos aspectos relacionados à cultura, comportamentos, atitudes, respostas, assim de como ocorre a organização médica e a assistência institucional à saúde em seus lócus conforme aponta Rosemberg em seu referencial ${ }^{6}$.

Tais agravantes têm colocado o Brasil próximo a ser o novo epicentro da doença ${ }^{27}$, no qual vivenciamos durante o auge do contexto pandêmico, a presença de atos fraudulentos e corruptos em processos licitatórios para a construção de hospitais de campanha, em compras de materiais médicos e hospitalares, em repasses de recursos financeiros para a contratação de equipe profissional de saúde e no fornecimento de auxílio emergencial para pessoas em situação de elevada vulnerabilidade, e que foram impactadas seve- ramente pela determinação da quarentena e do distanciamento social ${ }^{28-31}$.

Além desses obstáculos, outros desafios com o foco na subsidiação das políticas de enfrentamento à pandemia e dos impactos gerados pela COVID-19 no Brasil são evidenciados, como a ampliação da capacidade dos sistemas de informação e de testagens de amostra da população, a formulação adequada de indicadores em saúde e a condução e suspensão gradual do isolamento ${ }^{1}$.

$\mathrm{O}$ momento pandêmico exige das autoridades responsabilidade, comprometimento e transparência na resolução dos problemas que emergem diariamente. $\mathrm{O}$ discurso revelou crescentes preocupações com relação a um possível colapso no sistema de saúde no sentido de não oferecer condições adequadas para um suporte às pessoas afetadas pela COVID-19. Os homens temem que o Brasil vivencie um cenário desfavorável como visto em outros países. Essa é uma preocupação de quase todos os países atingidos pela doença, em especial aqueles com maior fragilidade no sistema de saúde. No Brasil, o discurso masculino desvela preocupação ainda maior, ao considerar as desigualdades e a grande expansão demográfica e territorial do país, que pode se configurar em fatores complicantes.

Países como a Itália, a Espanha, o Reino Unido e os EUA estão sendo devastados pela doença, nos quais os sistemas de saúde não respondem de modo satisfatório. Por outro lado, a Alemanha apresentou um cenário favorável evidenciado pelo achatamento da curva mediante medidas de prevenção realizadas coletivamente. Já no Brasil, estados como Amapá, Ceará, Manaus, Pará, Rio de Janeiro e São Paulo, já sofrem com o colapso no sistema de saúde ${ }^{5}$.

Por consequência da quarentena e do distanciamento social determinadas no país, as preocupações geradas nos homens estão fincadas na insegurança e no receio da ruptura das interações de sociabilidade afetiva e familiar anteriormente estabelecidas. Desvelou-se também preocupações na subsistência e em cumprir o seu papel de provedor. Com a quebra de vínculos a relação de proximidade que o contato físico proporciona, revela prejuízos à rede de interação, apoio e/ou suporte social. Em pandemias anteriores, o enquadramento da doença é socialmente negociado a fim de garantir o enfrentamento e a superação da doença epidêmica, o que inclui a pandemia em um sistema interativo, invadindo diversas esferas das manifestações da vida humana ${ }^{6}$.

A ruptura do cotidiano de homens no Brasil gera a perda da espontaneidade, desconfortos, insegurança e instabilidade, potencializa 
as incertezas e a percepção da incapacidade de prever a evolução da doença e retomada de suas atividades. Desse modo, ao cogitarem o prolongamento do tempo de distanciamento social os homens expõem seus conflitos afetivos, conjugais e dificuldade para manutenção dos vínculos. A negociação para a adesão de novos hábitos, em especial o afastamento de pessoas de seu convívio, têm repercutido no aumento expressivo de distúrbios psico-emocionais, danos autoinfligidos, uso abusivo de substâncias e violência intrafamiliar ${ }^{32-36}$.

A negação da gravidade da doença e dos seus impactos é um padrão que se repete na construção da explicação da doença por parte dos homens neste estudo e colabora para ampliar o problema e impedir que o processo avance para o segundo ato, no qual se espera uma aproximação do fenômeno, novas representações e percepções formuladas em torno destas experiências a fim de lidar com os desafios advindos da pandemia ${ }^{6}$.

A aceitação, elemento do segundo ato, é forjada na aproximação e no conhecimento de mortes de pessoas conhecidas ou próximas de onde residem. No entanto, aquelas mortes vistas em noticiários fazem parte do primeiro ato, possibilitando a revelação progressiva e a apropriação do conhecimento sobre a doença. Nesse sentido, ao constatar o aumento da mortalidade no Brasil, os homens se percebem atingidos mentalmente, com ameaças ao seu bem-estar psicológico, temem o adoecimento mental, motivados em grande parte pelo confinamento, desassossego, angústia, ansiedade e pelo estresse pós-traumático $^{37-40}$.

Ao serem impossibilitados de transitar livremente nos espaços de sociabilidade, os homens colaboram para o enquadramento da doença elaborando representações que se ancoram no sentimento de impotência, e na percepção da própria incapacidade de ajudar as pessoas que se encontram afetadas pela COVID-19, em especial aquelas em vulnerabilidade que vivem ao seu entorno. Esse sentimento de impotência também está associado ao medo e ao pânico frente ao desconhecido, e consequentemente paralisa o sujeito reduzindo sua capacidade de enfrentamento ${ }^{39}$.

O quarto ato descrito por Rosemberg, consiste em alcançar a reflexão, o reconhecimento da própria vulnerabilidade e a mudança da cultura de cuidados, assumindo assim uma consciência sanitária. A dificuldade de alcançar essa mudança a partir da conscientização e da própria experiência está relacionado às limitações dos cenários de vida, vivências no trabalho, acesso a cuidado e assistência à saúde, e dificuldades imposta pela sobreposição de iniquidades, aniquilações, principalmente nas populações de pessoas pretas e pobres $^{6}$, fundamentada na necropolítica ${ }^{41}$ e no contexto político e sócio-histórico das pandemias $^{6}$.

Vale ressaltar, que no Brasil assim como em outros países, as populações mais vulneráveis têm sido deterioradas pelos efeitos destrutivos da pandemia, que por sua vez, potencializam desigualdades, exclusões e iniquidades sociais, sendo este um desafio para a segurança global ${ }^{7}$. Teme-se ainda a elevação da fome e da miséria, como no caso dos homens em situação de rua, desabrigados, em situação carcerária, refugiados e imigrantes. Por essas razões, é crucial que sejam estabelecidas medidas de reparação pós-pandêmica ${ }^{42,43}$, bem como a revisão de modelos hegemônicos de masculinidades quee comprometam a compreensão masculina a respeito dos processos saúde e doença, da adoção de práticas de cuidado à saúde e a redução das vulnerabilidades por esse público ${ }^{12,44}$, uma vez que as masculinidades têm se apresentado como um marcador relevante de análise em fenômenos sócio-históricos em saúde, por se apresentar de maneira interseccionada, que tornam os homens vulneráveis e mais afetados pelas condições impostas pela pandemia.

É previsível que em função das experiências vividas pelos homens no curso da pandemia no Brasil, em que há a transição do fenômeno pandêmico, o surgimento de novos elementos dê conta de enquadrar a Covid-19 a partir dos atos característicos próprios. Nesse sentido, recomenda-se o aprofundamento das investigações sobre os atos no período de finalização da pandemia assim como a fase pós-pandêmica.

Por fim, os discursos dos homens expressaram de forma muito incipiente elementos de aceitação, os quais estariam diretamente vinculados à percepção de vulnerabilidade e justificativa para práticas de autocuidado. Não aceitar a doença, pode estar relacionado ao "não se sentir vulnerável”. Diferenças significativas de crenças e atitudes quanto ao gênero na pandemia da Covid-19 já foram levantadas em estudo brasileiro que aponta que as mulheres se percebem mais vulneráveis ao coronavírus e, por isso, expressam maior senso de autocuidado ${ }^{25}$. Nesse sentido, o enquadramento da doença pode evidenciar diferenças significativas de gênero que ainda foram pouco exploradas.

Reconhecemos limitações nesta pesquisa. A principal delas, reside no método de produção de dados que restringe a construção da narrativa so- 
bre a doença e não permite aprofundamento de aspectos como nos encontros face a face.

\section{Conclusão}

Os sentimentos de homens explorados neste estudo, evidenciam que o enquadramento da doença Covid-19 no Brasil se encontra em evolução e delimitada em três atos, seu completo enquadre desse fenômeno ainda é incipiente no que se refere ao quarto ato.

Os achados implicam a publicização de subsídios essenciais para o avanço no conhecimento sobre a doença no país, como forma de implementar ações e políticas públicas em saúde que sejam compatíveis com as demarcações apresentadas sócio-historicamente pela população, garantindo em especial, a coerência, a resolubilidade, a especificidade e a singularidade.

\section{Colaboradores}

AR Sousa: concepção do estudo, coleta, análise e interpretação dos dados, redação e correção final; ESS Carvalho e TS Santana: análise e interpretação dos dados, redação e correção final; AFL Sousa: interpretação dos dados e redação crítica; TFG Figueiredo: coleta dos dados e redação; OJV Escobar e TN Mota: interpretação dos dados e redação crítica e A Pereira: interpretação dos dados, redação final. 


\section{Referências}

1. Barreto ML, Barros AJD, Carvalho MS, Codeço CT, Hallal PRC, Medronho RA, Struchiner CJ, Victora CG, Werneck GL. O que é urgente e necessário para subsidiar as políticas de enfrentamento da pandemia de COVID-19 no Brasil? Rev bras epidemiol 2020; 23:e200032.

2. Organização Pan-Americana de Saúde (OPAS), Organização Mundial da Saúde (OMS). Folha informativa - COVID-19 (doença causada pelo novo coronavírus). 2020. [cited 2020 May 9]. Available from: https:// www.paho.org/bra/index.php?option $=$ com_conten$\mathrm{t} \&$ view $=$ article\&id $=6101$ : covid19\&Itemid $=875$

3. United Nations (UN). Lança plano para 'derrotar o vírus e construir um mundo melhor'. [cited 2020 May 9]. Available from: https://nacoesunidas.org/onu-lanca -plano-para-derrotar-o-virus-e-construir-um-mundo-melhor/

4. United Nations (UN). Dos Objetivos de Desenvolvimento do Milênio aos Objetivos de Desenvolvimento Sustentável. [cited 2020 May 10]. Available from: https://www.br.undp.org/content/brazil/pt/home/ presscenter/articles/2015/12/07/dos-objetivos-de -desenvolvimento-do-mil-nio-aos-objetivos-de- desenvolvimento-sustent-vel.html

5. Brasil. Ministério da Saúde (MS). Secretaria de Vigilância em Saúde. Doença pelo coronavírus 2019. Boletim Epidemiológico 17 - COE Coronavírus - 09 de abril de 2020. Brasília: MS; 2020. [cited 2020 May 9]. Available from: https://www.saude.gov.br/images/ pdf/2020/May/29/2020-05-25---BEE17---Boletimdo-COE.pdf

6. Pickstone JV. Explaining epidemics and other studies in the history of medicine. Med Hist 1993; 37(4):453454.

7. Rosenberg C, Mantovani R. On the history of medicine in the United States, theory, health insurance, and psychiatry: an interview with Charles Rosenberg. Hist Cienc Saude Manguinhos 2016; 23(1):211-220.

8. Nicola M, Alsafi Z, Sohrabi C, Kerwan A, Al-Jabir A, Losifidis C, Agha M, Agha R. The socio-economic implications of the coronavirus pandemic (COVID -19):A review. Int J Surg 2020; 78:185-193.

9. Quinn SC, Kumar S. Health inequalities and infectious disease epidemics: a challenge for global health security. Biosecur Bioterror 2014; 12(5):263-273.

10. Fundação Oswaldo Cruz (Fiocruz). Impactos sociais, econômicos, culturais e políticos da pandemia. 2020. [cited 2020 May 9]. Available from: https://portal. fiocruz.br/impactos-sociais-economicos-culturais-e -politicos-da-pandemia.

11. Estrela FM, Soares CF, Cruz MA, Silva AF, Santos JRL, Moreira TMO, Lima AB, Silva MG. Pandemia da covid 19: Refletindo as vulnerabilidades a luz do gênero, raça e classe. Cien Saude Colet. 2020.

12. Gomes R, Couto MT, Keijer B. Hombres, género y salud. Salud Colectiva 2020; 16:2788.
13. Purdie A, Hawkes S, Buse K, Onarheim K, Aftab W, Low N, Tanaka S. Sexo, gênero e COVID-19: Dados desagregados e disparidades de saúde. 2020. [cited 2020 May 9]. Available from: https://blogs.bmj.com/bmjgh/2020/03/24/sex-gender-and-covid-19-disaggregated-data-and-health-disparities/

14. The guardian [site da internet]. Os homens têm muito mais probabilidade de morrer de coronavirus - mas por quê? Reino Unido, 26 abr. 2020. [cited 2020 May 9]. Available from: https://www.theguardian.com/ world/2020/mar/26/men-are-much-more-likely-todie-from-coronavirus-but-why.

15. Schurz H, Salie M, Tromp G, Hoal EG, Kinnear CJ, Möller M. The X chromosome and sex-specific effects in infectious disease susceptibility. Hum Genomics 2019; 13(1):2.

16. Sousa AR, Queiroz AM, Florencio RMS, Portela PP, Fernandes JD, Pereira A. Homens nos serviços de atenção básica à saúde: repercussões da construção social das masculinidades. Rev Bai Enf 2016; 30(3):110.

17. Sousa AR. How can COVID-19 pandemic affect men's health? a sociohistoric analysis. Rev Pre Infec e Saúde 2020; 6:10549.

18. Freitas MTA. A abordagem sócio-histórica como orientadora da pesquisa qualitativa. Cad. Pesqui. 2002(116):22-32.

19. Padilha MI, Bellaguarda MLR, Sioban N, Maia AR, Camargo CR. O uso das fontes na condução da pesquisa histórica. Texto contexto - enferm 2017; 26(4):e2760017.

20. Biernacki P, Walford D. Snowball sampling: Problems and techniques of chain referral sampling. Social Meth Res 1981(2):141-163.

21. Fontanella BJB, Magdaleno R. Saturação teórica em pesquisas qualitativas: contribuições psicanalíticas. Psicol Estudo 2012; 17(1):1763-1771.

22. Lefevre F, Lefevre AMC. O sujeito coletivo que fala. Comunic Saúde Educ 2006; 10(20):517-524.

23. Lefevre F, Lefevre, AMC, Marques, MCC. Discurso do Sujeito Coletivo, complexidade e auto-organização. Cien Saude Colet 2009; 14(4):1193-1204.

24. Lancet. COVID-19 in Brazil: "So what?". lancet 2020; 395(10235):1461.

25. Lima DLF, Dias AA, Rabelo RS, Cruz ID, Costa SC, Nigri FMN, Neri JR. COVID-19 no estado do Ceará, Brasil: comportamentos e crenças na chegada da pandemia. Cien Saude Colet 2020; 25(5):1575-1586.

26. Brasil. Ministério da Saúde (MS). Coronavírus: Boletim epidemiológico 15. Brasília: MS; 2020. [cited 2020 May 9]. Available from: https://portalarquivos.saude. gov.br/images/pdf/2020/May/09/2020-05-06-BEE15-Boletim-do-COE.pdf

27. CNN. [site da internet] Como evitar que o Brasil se torne o novo epicentro da Covid-19?. 2020. [cited 2020 May 9]. Available from: https://www.cnnbrasil.com. br/saude/2020/05/09/como-evitar-que-o-brasil-setorne-o-novo-epicentro-da-covid-19 
28. A Tribuna. [site da internet]. 73.2 mil militares recebem auxílio emergencial indevidamente e devem ser punidos. Artigo jornalístico. [cited 2020 May 9]. Available from: https://www.atribuna.com.br/noticias/atualidades/73-2-mil-militares-recebem-aux\%C3\%ADlio-emergencial-indevidamente-e-devem-ser -punidos-1.100799

29. O Globo. [site da internet]. Aquisição de hospitais de campanha tem proposta plagiada e 'concorrente' fantasma no RJ. Artigo jornalístico. [cited 2020 May 9]. Available from: https://g1.globo.com/rj/rio-de-janeiro/noticia/2020/04/17/apos-reportagem-do-g1-witzel-manda-apurar-indicio-de-fraude-na-compra-de -hospitais-de-campanha.ghtml

30. UOL. [site da internet]. Coronavírus: hospital vitima de fraude pediu doaçoses para combater covid-19. Artigo jornalístico. [cited 2020 May 9]. Available from: https:/noticias.uol.com.br/saude/ultimas-noticias/ redacao/2020/04/15/hospital-pivo-de-operacao-doacoes-combater-coronavirus-fraude-mascara.htm

31. Santos IS, Vieira FS. Direito à saúde e austeridade fiscal: o caso brasileiro em perspectiva internacional. Cien Saude Colet 2018; 23(7):2303-2314.

32. Alradhawi M, Shubber N, Sheppard J, Ali Y. Effects of the COVID-19 pandemic on mental well-being amongst individuals in society- A letter to the editor on "The Socio-Economic Implications of the Coronavirus and COVID-19 Pandemic: A Review”. Int J Surg 2020; 78:147-148.

33. Ahmed Z, Ahmed O, Aibao Z, Hanbin S, Siyu L, Ahmad A. Epidemic of COVID-19 in China and Associated Psychological Problems. Asian J Psychiatr 2020; 51:102092.

34. Marques ES, Moraes CL, Hasselmann MH, Deslandes SF, Reichenheim ME. Violence against women, children, and adolescents during the COVID-19 pandemic: overview, contributing factors, and mitigating measures. Cad Saude Publica 2020; 36(4):e00074420.

35. Vieira PR, Garcia LP, Maciel ELN. The increase in domestic violence during the social isolation: what does it reveals? Rev bras epidemiol 2020; 23:200033.

36. Campbell AM. An increasing risk of family violence during the Covid-19 pandemic: Strengthening community collaborations to save lives. Forensic Science Inter 2020; 2:100089.

37. Peçanha T, Goessler KF, Roschel H, Gualano B. Social isolation during the covid-19 pandemic can increase physical inactivity and the global burden of cardiovascular disease. Am J Physiol Heart Circ Physiol 2020; 318(6):H1441-H1446.

38. Berg-Weger M, Morley JE. Loneliness and Social Isolation in Older Adults During the Covid-19 Pandemic: Implications for Gerontological Social Work. $J$ Nutr Health Aging 2020; 24(5):456-458.

39. Ornell, F, Schuch, JB, Sordi, AO, Kessler, FHP. "Pandemic fear" and COVID-19: mental health burden and strategies. Braz J Psychiatry. 2020; 42(3):232-235.
40. Holmes, E, O'Connor R, Perry VH, Tracey I, Wesselu S, Arseneault L, Ballard C, Christensen H, Silver RC, Everall I, Ford T, John A, Kabir T, King K, Madan I, Michie S, Przybylski AK, Shafran R, Sweeney A, Worthman CM, Yardley L, Cowan K, Cope C, Hotopf M, Bullmore E. Multidisciplinary research priorities for the COVID-19 pandemic: a call for action for mental health science. Lancet Psychiatry 2020; 7(6):547-560.

41. Mbembe A. Necropolitica: biopoder soberania estado de exceção política da morte. São Paulo: N-1 edições; 2018.

42. Horton R. Offline: CoHERE-a call for a post-pandemic health strategy. Lancet 2020; 395(10232):1242.

43. Brooks SK, Webster RK, Smith LE, Woodland L, Wessely S, Greenberg N, Rubin GJ. The psychological impact of quarantine and how to reduce it: a quick review of the evidence. Lancet 2020; 395(10227):912920.

44. Connell, RW, Messerschmidt, JW. Masculinidade hegemônica: repensando o conceito. Rev Estud Fem 2013; 21(1):241-282.
Artigo apresentado em 19/05/2020

Aprovado em 16/06/2020

Versão final apresentada em 18/06/2020 
